\title{
GRÈCE ANCIENNE, GRËCE MODERNE DANS L'INTELLIGENTSIA FRANÇAISE DE 1797 À 1832
}

Lorsque, sous la Révolution, Adamantios Coray, savant grec vivant en France, va chercher un passeport à la Convention, l'annonce de sa nationalité, raconte-t-il, jette la stupeur dans l'Assemblée. Certains Conventionnels iront jusqu'à le toucher pour s'assurer qu'un Grec peut être un homme fait de chair et de sang. La proximité avec l'Antiquité grecque est telle, que l'on ne peut alors imaginer qu'il existe une Grèce moderne, peuplée de Grecs bien vivants. C'est en 1797 que la France révolutionnaire rencontre la Grèce moderne. Le 28 juin, le général Gentilly débarque avec ses troupes dans les îles Ioniennes, à Corfou, au milieu de l'allégresse des habitants. Le général Buonaparte nous raconte comment il est accueilli par le Papa, chef religieux orthodoxe qui lui dit :

"Français, vous allez trouver dans cette île un peuple ignorant dans les sciences et les arts qui illustrent les nations; mais ne le méprisez pas pour cela; il peut devenir encore ce qu'il a été : apprenez, en lisant ce livre, à l'estimer "'

Ce livre, c'est l'Odyssée d'Homère.

Le roman de Stendhal, Armance, met en scène un jeune noble, Octave de Malivert. "Jeune cœur [...] qui se trouvait en contradiction avec les événements de la vie réelle ", Octave est singulier par rapport à son époque, la Restauration, et à son milieu. Son amour pour Armance devenu impossible, il justifie son départ par le désir d'aller combattre aux côtés des Grecs modernes soulevés depuis 1821 contre l'occupant turc. Octave trouve ainsi dans le roman à s'inscrire, plus que tous les autres personnages, dans la réalité de son temps, de cette année 1827 où le tour dramatique pris par la guerre de Grèce retient l'attention du public européen. Premier roman de Stendhal, Armance apparaît comme une prise de

1. Le rapport du général Buonaparte, du 14 thermidor ( $1^{\text {er }}$ août 1797$)$, publié dans $L e$ Moniteur du 21 ( 8 août) est cité par Pierre VIDal-Naquet dans sa préface aux Mémoires du général Makriyannis, trad. Denis KoHLER, Paris, Albin Michel, 1986, et dans « La place de la Grèce dans l'imaginaire des hommes de la Révolution ", in ID., La Démocratie grecque vue d'ailleurs, Paris, Flammarion, 1990.

Revue de synthèse : IV S. No 4, oct.-déc. 1990. 
possession de l'Histoire, dans la recherche d'une forme moderne d'écriture capable de mettre en œuvre le réel et de le dire ${ }^{2}$. Le geste de l'écrivain s'accomplit dans une sacralisation de l'espace et du temps: la recherche s'inscrit dans une perspective symbolique et mythologique. L'écriture s'empare du mythe et l'intériorise. Octave meurt, couché sur le pont d'un navire en vue des côtes de la Grèce :

« Un mousse du haut de la vigie cria : Terre! C'était le sol de la Grèce et les montagnes de Morée que l'on apercevait à l'horizon. Un vent frais portait le vaisseau avec rapidité. Le nom de la Grèce réveilla le courage d'Octave : je te salue, se dit-il, ô terre des héros! Et à minuit, le 3 mars, comme la lune se levait derrière le mont Kalos, un mélange d'opium et de digitale préparé par lui délivra doucement Octave de cette vie qui avait été pour lui si agitée. Au point du jour, on le trouva sans mouvement sur le pont, couche sur quelques cordages. Le sourire était sur ses lèvres, et sa rare beauté frappa jusqu'aux matelots charges de l'ensevelir ${ }^{3}$.

Dans le mythe grec, Thésée (ou mot à mot " l'homme couché ») vogue vers sa patrie, Athènes. Il oublie de faire le signe de reconnaissance. Dans Armance, c'est la fausse lettre de la jeune fille, laissée dans la caisse d'oranger qui sert de boîte aux lettres aux jeunes gens, qui joue ce rôle de fatalité, que le silence d'Octave - absence de signes - renforce encore. Sur les côtes de Grèce, au lieu le plus élevé d'Athènes, Égée, le père, voit le vaisseau à voile noire - signe de mort - poindre à l'horizon, et se jette dans la mer. De la terre - «Terre !" - à l'ensevelissement - dans la mer? comme une image en négatif, le roman vient se superposer au mythe. Le renversement de la mort d'Égée à la mort d'Octave dit la réalité du mythe, dans la création d'une réalité romanesque, création mythologique, réalité de l'histoire ${ }^{4}$. Dans la perspective symbolique et mythologique de saisie du réel, du présent, le ressourcement semble effectivement passer par la Grèce ancienne : elle est source, inspiration, mère. Mais quelle est cette Grèce, profondément enracinée dans les mémoires, vers laquelle on se tourne comme en dernier recours pour recoller à l'Histoire et retrouver l'unité du Moi brisée par les événements?

2. Le sous-titre du roman, ou quelques scènes d'un salon de Paris en 1827, indique, par la précision de la date et du lieu, que l'action se situe dans la réalité contemporaine (l'ouvrage parait en 1827), d'un temps et d'un espace définis. C'est seulement à partir de cette saisie du réel, de cette suspension du temps que la parole ou l'ecriture devient possible, et par là mème, le jeu sur l'Histoire.

3. Stendhal, Amance, Paris, Garnier-Flammarion, 1967, p. 192.

4. Le roman fait jouer le temps sur l'espace, 1827 et l'ailleurs grẹc comme miroir réflèchit le passé dans un présent - celui de la mort? La masse du mont Kalos sous la lune nous renvoie à la beauté du corps inerte d'Octave à l'aube. L'espoir de la "belle mort " de l'hoplite grec devient, dans la douceur souriante de la délivrance d'Octave, parti sous le prétexte d'aller combattre en Grèce, le seul espoir d'être un "beau mort ". L'action, en perdant sa féminité ou son féminin, se fait, dans Stendhal, individuelle et solitaire. 
Le concours de peinture des Prix de Rome, par le choix des sujets, nous donne une idée assez juste de l'image de la Grèce antique au début du $\mathrm{xIx}^{\mathrm{e}}$ siècle et de son importance. Sur les 38 sujets de Composition historique proposés aux candidats de 1797 à 1834,27 sont "grecs " ${ }^{5}$. Ils sont de plusieurs types : Grèce homérique, de l'Iliade et de l'Odyssée ${ }^{6}$; Grèce des Tragiques, Eschyle, Sophocle, Euripide ${ }^{7}$; Grèce des dieux et des héros ${ }^{8}$; Grèce, enfin, des grands personnages, semi-légendaires, figures de proue de l'archétype démocratique grec ${ }^{9}$. L'image claire et limpide de la Grèce ancienne que nous renvoient les arts, rejoint cette Antiquité classique dispensée par l'éducation, se référant toujours aux mêmes textes, aux mêmes personnages privilégiés ${ }^{10}$. La rhétorique, pédagogie morale pour une éducation morale, s'appuie sur une Antiquité de morceaux choisis. Mais l'identification des révolutionnaires au modèle antique a invalidé toute possibilité d'imitation. Il faut alors trouver une autre voie pour appréhender la Grèce, changer de registre pour pouvoir à nouveau y avoir recours. Une autre Antiquité se dessine, une autre lecture de ces mêmes textes, lus et relus, est possible.

C'est ce que montre Charles Nodier dans Smarra ou les démons de la nuit. Le conte est sous-titré dans l'édition originale de 1821 «songes romantiques traduits de l'esclavon du comte Maxime Odin ". Si ce titre donne l'idée d'un ouvrage influencé par le séjour de Nodier en Illyrie et écrit sur le mode de la littérature frénétique (inspiré des romans noirs anglais), il ne faut pas retenir ce seul aspect. La phrase d'André Chénier - «Sur des sujets nouveaux, faisons des vers antiques » - qui ouvre la nouvelle préface donnée au conte en 1832, nous éclaire sur le sens de la recherche menée par Nodier dans Smarra, celle du modèle grec. Le travail de la traduction mis en cuvre par l'auteur s'exerce non pas sur un

5. C'est en 1797 que le concours est rétabli. Il faut noter que les quatre premiers sujets et ils prolongent en cela les références antiques des révolutionnaires - ont trait à Rome. 1797, "La mort de Caton d'Utique " d'après Plutarque; les trois suivants sont inspirés de Tite-Live : 1798, "Le combat des Horaces et des Curiaces "; 1799, " Manlius Torquatus condamne son fils à mort "; 1800, " Antiochus renvoie son fils à Scipion "; cf. Les Concours des Prix de Rome 1797-1863, Catalogue par Ph. Grunchec, Paris, E.N.S.B.A., 1986.

6. "Achille recevant les ambassadeurs d'Agamemnon" (1801); "Priam aux pieds d'Achille " (1809)... « Ulysse et Télémaque massacrant les prétendants " (1812)... En 1834, " Homère chantant ses vers ", le sujet était inspiré d'écrits d'André Chénier.

7. "Oreste et Pylade investis par les bergers " (1822)... « Antigone donnant la sépulture à Polynice " (1825)...

8. "Thésée vainqueur du Minotaure" (1807); "Castor et Pollux délivrant Hélène" (1817)...

9. Phocion (1804), Démosthène (1805), Diagoras (1814), Thémistocle (1819), Alcibiade (1824)...

10. L'ouvrage de l'abbé Jean-Jacques Barthélemy, Le Voyage du jeune Anacharsis en Grèce, dans le milieu du $N^{e}$ siècle avant l'ère vulgaire, 4 vols, Paris, 1788, en est un bon exemple. Le grand nombre de rééditions du Voyage témoigne de son influence dans la vision de la Grèce classique. 
texte moderne, en esclavon, mais sur les textes de l'Antiquité classique. Le conte, " écrit sous l'inspiration de l'Antiquité la plus pure ", est une étude, un pastiche, ou plutôt les traductions mises bout à bout des phrases les plus intraduisibles des Anciens et de quelques modernes ${ }^{11}$. Le travail sur la langue française (à partir du grec et du latin) se fait dans la perspective de l'écriture "fantastique " du rêve ou du cauchemar. Les sujets inspirés par la Grèce ne paraissent pas épuisés à Nodier. L'Ane d'or d'Apulée servira ainsi de canevas au conte. Passant par l'étrange Thessalie, et la mythologie des peuples slaves (« Smarra " signifie cauchemar), la lecture par le rêve de Nodier découvre une nouvelle compréhension de l'Antiquité. La face cachée ou mystérieuse du monde classique peut être révélée : "L'Odyssée d'Homère est du fantastique sérieux " 12 . La «fantaisie " est l'âme de la création des temps antiques. Son caractère, comme en témoigne Homère, est la naïveté, impossible à " notre siècle incrédule ", constate Nodier. Dans la voie du fantastique, envisagée par l'auteur, la fantaisie des temps modernes résidera dans la création de "types extraordinaires mais possibles ", comme les caractères du rêve de l'homme de la nuit.

Ce que nous apprend Nodier avec Smarra, c'est qu'entre l'Antiquité grecque et la France du début du XIx ${ }^{\complement}$ siècle, l'Histoire s'est intercalée. Aux mythologies de la Grèce païenne vient se superposer une mythologie moderne, nationale, qui prend ses racines dans le sol même du pays. La France, l'Europe, sont chrétiennes. Entre les Grecs anciens et les penseurs de la France moderne, on ne parle plus d'une filiation mais bien plutôt d'une communauté, celle des créateurs. Le christianisme oblige à une relecture de l'Histoire qui trouve son origine dans le xvir siècle augustinien ${ }^{13}$. Avec Pascal, avec Racine, ce qui se présentait comme une

11. Cf. préf. de 1832, in Charles Nodier, Contes. Smarra, Trilby et autres contes, Paris, Flammarion, coll. « Garnier-Flammarion », 1980, p. 76-78 : « Sauf quelques phrases de transition, tout appartient à Homère, à Théocrite, à Virgile, à Catulle, à Stace, à Lucien, à Dante, à Shakespeare, à Milton. [...] J'avais tellement redouté de me mesurer avec la haute puissance d'expression qui caractérise l'Antiquité que je m'étais caché sous le rôle obscur de traducteur." Le camouflage fut complet pour les lecteurs de 1821 et Smarra qualifiée de livre " romantique ".

12. Ibid., p. 73-74. Rappelons sur cette piste de l'émergence d'un aspect mystérieux, souterrain - dans le sens d'occulte - de l'Antiquité classique, que Nodier a fait partie d'une secte pythagoricienne, « Les Méditateurs ", fondée par Maurice Quail. Nodier écrira en souvenir de cet engagement Apothéose et Imprécations de Pythagore (1808).

13. Le xvi ${ }^{e}$ siècle redécouvre saint Augustin (passant outre la tradition se référant à saint Thomas) et l'interprète. Il s'opère ainsi une sorte de synthèse dans les écrits des hommes du Grand Siècle entre l'augustinisme (saint Augustin consomme la rupture entre monde grec et monde chrétien, inscrit le christianisme comme un aboutissement, la Providence est conque comme la fin de l'action divine) et la tradition médiévale. Saint Augustin l'Africain a baigné dans l'atmosphère du néo-platonisme dont il parle dans Les Confessions. 
juxtaposition, une coordination sans aucun sens (autrement que dans une relation d'archétype ou de modèle "à la manière de... ", agrégat de références) devient disjonction, puis une synthèse qui donne un sens à l'Histoire. L'Histoire est finalisée ${ }^{14}$. Le sens s'organise autour d'un point initial qui détermine un en deçà et un au-delà. En deçà, tout annonce : Adam et les prophètes sont des personnages historiques. Nabuchodonosor est un serviteur de Dieu pour Bossuet.

Si l'on évoque ainsi la lecture de l'Antiquité telle qu'elle s'est élaborée au Xvir siècle pour comprendre le rapport à l'Antique tel qu'il s'établit au début du $\mathrm{xIx}^{\mathrm{e}}$ siècle, c'est que la filiation à la Grèce passe effectivement par le relais de cette tradition chrétienne, contre la philosophie rationaliste des Lumières ${ }^{15}$. La référence aux textes du Grand Siècle est manifeste à cette époque. Elle est perceptible tant dans les travaux d'un érudit historien comme le baron de Sainte-Croix ${ }^{16}$ que dans les écrits de la grande figure du début du XIX siècle, François-René de Chateaubriand. Rompant avec le Siècle des Lumières ${ }^{17}$, Chateaubriand peut tout à la fois se référer à Pascal et à Homère. La perspective ouverte par la lecture chrétienne de l'Antiquité au Xvil siècle, dans le cadre d'une pensée de la liberté de l'individu (Descartes, Pascal), est celle d'une vérité morale de l'histoire, et

14. Michel Serres, Le Système de Leibniz et ses modèles mathémariques, Paris, P.U.F., 1968, « Le Paradigme pascalien », t. 2, p. 710 : Pascal « organise et constitue le temps. L'histoire du salut est finalisée, ou plus généralement, l'histoire a un sens, une direction privilégiée ou définissable que n'a plus l'espace de nos expériences scientifiques. Laissant le monde pour l'histoire, désormais munie de cette référence, Pascal exprime l'essence des religions dites modemes, par opposition aux cosmiques traditionnelles, qui avaient laissé des traces jusqu'à lui, dans la sienne propre. Il est ainsi à la fois l'héritier de la découvere judazque de la monodromie du temps dont il fixe la finalité, et le préurseur des philosophies modemes de thistoire comme acoomplissement progressif d'une instance, quelle qu'efte soit".

15. Les diffèrents mouvements du début du $x x^{\varepsilon}$ sjècle : néo-platonisme (Platon penseur chrétien), néo-christianisme (comme celui des Martyrs) quj déboucbe sur un chnistianisme social (individu dans la société, problème de sa liberté, of. Lamennais, par ex.) etc., s'ils afinrment prendre leur source dans l'Antiquité, apparaissent médjatisés par la pensée classique.

16. Le baron de Sainte-Croix (1746-1809) peut être un bon exemple de ce glissement des références et du refus des Lumières aftiché après la Révolution. Avant les événements de 1793, SAINIE-CroIX se présente, dans son Examen critique des anciens historiens d'Alexandre le Grand (1775), comme un homme épris de liberté ; un érudit s'attachant à ériger sa matière, l'histoire, en une "science des faits » en référence au modèle des sciences exactes (newtonien), notamment la chimie de Lavoisier (1772). Au début du $x \times x$ siècle, la position est totalement bouleversée; l'Examen critique réecrit en 1804 en témoiqge. L'histoire est désormais comprise comme un art ( $"$ art historique $")$ dont les principes moraux - pour une vérité morale de l'histoire - sont explicitement tirés du xvir siècle : Boileau, Bossuet, Pascal, le cardinal de Retz. L'histoire est un produit de la Providence. Au lendemain de la Révolution, les convictions de Sainte-Croix sont d'ordre esthétique (le style) et moral (l'histoire comme enseignement).

17. Le Chateaubriand de l'autre monde dont parle Pierre Barberis, « Les réalités d'un ailleurs : Chateaubriand et le "Voyage en Amérique ", Littérature, 21, Paris, Larousse, févr. 1976, p. 91-103. L'Amérique découverte par Chateaubriand invalide « l'expérience philosophique " du xvil' siècle avec laquelle le voyageur était parti. L'ailleurs américain n'existe plus. Au héros des Natchez, "il ne restait d'abri que l'océan ". 
c'est bien en ce sens que travaillent les historiens du début du siècle ${ }^{18}$. Une synthèse s'opère entre monde grec et monde chrétien.

Le double mouvement d'une symbolique grecque et d'une symbolique chrétienne est ainsi à l'œuvre dans Les Martyrs (1809). L'auteur part en 1806 pour un voyage de près de deux ans en Grèce et en Orient à la recherche des paysages - «images " - des Martyrs. Le récit en sera L'Itinéraire de Paris à Jérusalem et de Jérusalem à Paris, en allant par la Grèce, et revenant par l'Égypte, la Barbarie et l'Espagne (1811). Le voyage en Grèce se fait dans la perspective de Jérusalem. Dans cet itinéraire qui mène le voyageur de Paris à Jérusalem et de Jérusalem à Paris, la Ville Sainte est autant aboutissement que point de départ. Voyage dans les « lieux saints » de l'histoire grecque, dans les Lieux Saints, berceau de la religion chrétienne, point historique initial pour le croyant, le pèlerinage est double et se traduit dans l'écriture d'un récit de voyage. Le terme d'itinéraire, terme positif, indique bien que le voyage - voyage réel et voyage symbolique - se traduit sur une carte, où des lignes vont relier entre eux les points qui marquent les étapes du pèlerinage religieux, donnant un sens à l'espace. Comme une empreinte sur une carte géographique, les traces de l'itinéraire ne s'effaceront pas. L'Itinéraire s'inscrit en contrepoint du voyage mythique de la fondation de Rome, en contrepoint du sillage des vaisseaux qui s'évanouit dans la mer ${ }^{19}$. Vou d'un croyant, il rejoue les pèlerinages du Moyen Âge, le départ d'un Joinville pour la croisade ${ }^{20}$. Le sens de l'Histoire naît dans ces équivalences, dans ces reconnaissances d'identité d'une époque à l'autre, sous le signe des Muses et du Moyen Âge. Chateaubriand est un croisé, croisé antique, " martyr de l'indépendance », croisé moderne, " martyr de la vérité " ${ }^{21}$; l'un ne dément pas l'autre. La croisade se prolonge. La Grèce mère est chrétienne, et elle ne peut trouver à s'inscrire que dans la perspective du christianisme ${ }^{22}$. Dans cet élan qui pousse Chateaubriand vers la Grèce, ce

18. Voir, par ex., l'article " Histoire " dans le Rapport de la classe d'histoire et de littérature ancienne de l'Institut à Napoléon (1808). Rééd. critique sous la dir. de François HarToG, Paris, Belin, 1989. L'un des auteurs n'est autre que Sainte-Croix. C'est la grande erreur du Siècle des Lumières que d'avoir cru en la seule raison; l'oubli de la morale a conduit aux égarements révolutionnaires.

19. Itinéraire de Paris à Jérusalem, Paris, Garnier-Flammarion, 1968 (cité par la suite comme Itinéraire), p. 57 : « Moi, voyageur obscur, passant sur la trace effacée des vaisseaux qui portèrent les grands hommes de la Grèce à l'Italie, j'allais chercher les Muses dans leur patrie. " Vision de l'écart, Chateaubriand suit les traces d'Énée le Troyen, Énée le Grec, mais en sens inverse. Il écrit son voyage, un livre qui est tout sauf une trace évanescente.

20. Itinéraire, p. 54.

21. Itinéraire, p. 60 : «Quel moyen de nommer Saint-Jason et Saint-Sosistrate, apôtres des Corcyréens, sous le règne de Claude, après avoir parlé d'Homère, d'Aristote, d'Alexandre, de Cicéron, de Caton, de Germanicus ? Et pourtant un martyr de l'indépendance est-il plus grand qu'un martyr de la vérité ? "

22. Ainsi en témoigne le mouvement de la conversion de Cymodocée, « fille de la Grèce » dans les Martyrs. 
qu'il découvre effectivement sur le sol grec provoque une fracture avec la vision de cette Grèce mythique idéale ; fracture entre la représentation de l'homme lettré et le contact avec la réalité du voyageur. L'épreuve traversée dans ce voyage se traduit en écrit qui, pour imposer son caractère d'évidence et de pérennité, joue dans le sens d'une affirmation de l'écrivain, de l'homme surtout ${ }^{23}$. L'arrivée en Grèce est de ce point de vue exemplaire. Jeu de transparences dans cette représentation, entre la fable (Pylos) et l'histoire (la Sphactérie de Thucydide), avec le mythe (" sur le chemin d'Homère »), la Grèce, ses lieux, ses ruines sont lisibles.

« Le 10, au matin j'étais sur le pont avant le lever du soleil. Comme il sortait de la mer, j'aperçus dans le lointain des montagnes confuses et élevées : c'étaient celles de l'Élide : il faut que la gloire soit quelque chose de réel, puisqu'elle fait ainsi battre le coeur de celui qui n'en est que le juge. A dix heures, nous passâmes devant Navarin, l'ancienne Pylos, couverte par l'île de Sphactérie : noms également célèbres, l'un dans la fable, l'autre dans l'histoire. A midi nous jetâmes l'ancre devant Modon, autrefois Méthone, en Messénie. A une heure, j'étais descendu à terre, je foulais le sol de Grèce, j'étais à dix lieues d'Olympie, à trente de Sparte, sur le chemin que suivit Télémaque pour aller demander des nouvelles d'Ulysse à Ménélas » (Itinéraire, p. 62).

Reprenant cet épisode, Chateaubriand décrira plus précisément l'arrivée à Modon. Contact brutal avec la réalité de la Grèce contemporaine, le premier «Grec » rencontré est un Turc. Le ton est donné, le voyage dans la patrie des Muses se fera sous le signe du désert et du silence, des ruines et de la mort. Les pierres sont muettes. Les Grecs que cherchait Chateaubriand sont définitivement morts ${ }^{24}$. La proximité idéale avec la Grèce se dissout. La rupture entre une Grèce mythique et une Grèce « réelle » est consommée. La Grèce moderne, pays marqué par le joug ottoman, est misérable, semblable à cet Orient dégénéré que découvre Volney à Alexandrie $^{25}$. Les descriptions de la Grèce de Chateaubriand nous ren-

23. Cf. Itinéraire, préface à la $1^{\text {re }}$ éd., p. 41 : « Je prie donc le lecteur de regarder cet Itinéraire moins comme un Voyage que comme les Mémoires d'une année de ma vie. " La phrase est encore répétée dans le corps de l'ouvrage, p. 132.

24. C'est ainsi qu'il répond à un Turc qui lui demande la raison de son voyage : “ Je voyageais pour voir les peuples, et surtout les Grecs qui étaient morts » (Itinéraire, p. 87).

25. Pierre Vidal-NaQuet, "Paris-Athènes et retour ", in Athenes, ville capitale, dir. Yannis Tsiomis, Athènes, Caisse des fonds archéologiques, 1985, p. 37 : « Il y découvre non un Orient des Origines, comme en cherchaient tant de ses contemporains, mais l'écrasante misère de ce que nous appellerions aujourd'hui une capitale du tiers-monde, de ce qu'on appelait au xIx $^{e}$ siècle un pays mûr pour la colonisation. " La rupture entre un Orient des Origines - Égypte mystérieuse - et l'Orient misérable découvert et décrit par Volney est évidente si l'on pense au Monde primitif analysé et comparé avec le monde moderne de Antoine Court de Gebelin, Paris, 1771-1782. 
voient aux descriptions de l'Égypte de Volney ${ }^{26}$. Mais l'Itinéraire reste, contrairement au Voyage en Égypte et en Syrie (1784) où Volney décrit méthodiquement et scientifiquement les faits (géographiques et politiques) tels qu'ils lui apparaissent ${ }^{27}$, un itinéraire de la lettre et non de la pierre. Il s'écrit, répète. Il est itinéraire de la trace. La réalité politique, l'occupation turque et la misère des Grecs sous leur joug, ne renvoie qu'à un état de fait. La Grèce rencontrée par Chateaubriand n'est pas historique, elle ne fait pas trace dans la mémoire des hommes. La Grèce contemporaine est, dans le mouvement de l'écriture et pour que demeure son caractère d'évidence, rejetée en Orient. Autrement dit, elle n'est plus la Grèce. Le déplacement géographique s'impose comme le seul moyen pour appréhender une quelconque réalité. Les Grecs modernes sont des barbares ${ }^{28}$.

Le soulèvement des Grecs contre l'occupant turc en 1821 va offrir la possibilité d'intégrer la Grèce moderne dans l'histoire contemporaine. Le courage des combattants grecs, le récit des massacres, des actions héroïques abondamment repris par les journaux français (plus particulièrement ceux de tendances libérales et ultras), pour un public attentif et passionné, font basculer la Grèce dans l'événement et l'actualité. Le problème qui se pose alors à un homme comme Chateaubriand, ou à ses lecteurs, est celui de savoir s'il faut faire coïncider l'idéal avec la réalité. Faut-il voir dans cette nouvelle Grèce une résurgence historique de la Grèce ancienne telle qu'elle est restée dans les mémoires? Le mythe trouve-t-il là à s'incarner dans les faits ?

En 1826, Chateaubriand commence la publication de ses Euvres complètes. Il écrit une nouvelle préface à l'Itinéraire, ouvrant ainsi à cette autre lecture dictée par les événements. Les lieux traversés par le voyageur de 1806, les hommes rencontrés prennent un nouveau sens. Ou reprennent-ils leur sens? Les cris et le fracas de la lutte des Grecs modernes ont brisé ce silence partout présent dans l'Itinéraire. Leurs hauts faits ont rempli les interstices de leur histoire et de leur terre : nouvelles villes, nouveaux lieux, nouveaus noms. Le récit de voyage de 1811 est devenu en 1826 un " ouvrage de circonstance", une "carte topo-

26. Cf., par ex., Itinéraire, p. 169-170 et Voyage en Egypte et en Syrie, Paris/La Haye, Mouton et Co, 1959, p. 26. Chateaubriand connait bien évidemment le Voyage de Volney, of. Itinéraire, p. 374.

27. L'Itinéraire comme les "Mémoires d'une année de ma vie" semble répondre au Voyage, à cette phrase qui sert d'exergue au livre: " J'ai pensé que le genre des voyages appartenait à l'Histoire et non aux Romans. "Le Voyage servira de guide lors de la campagne d'Égypte.

28. Cf. Itinéraire, p. 168 : « Les monuments grecs modernes ressemblent à la langue corrompue qu'on parle à Sparte et à Athènes : on a beau soutenir que c'est la langue d'Homère et de Piaton, un mélange de mots grossiers et de constructions étrangères trahit à tout moment les Barbares." 
graphique du théâtre de cette guerre sacrée ». Sorte d'instantané de l' " avant renaissance " où les faits et les lieux sont posés, prêts à prendre vie, l'Itinéraire marque un moment de suspension dans le temps et dans l'Histoire ; moment où tout « n'était pas encore » ${ }^{29}$. François-C.-H. Pouqueville, consul de France à Jannina auprès du fameux Ali Pacha, publie en 1824 à Paris son Histoire de la régénération de la Grèce, historique du réveil grec de 1740 à 1824 . Il mêle dans son récit la Grèce de l'histoire ancienne et cette Grèce qui inscrit encore, au moment où il publie son ouvrage, ses événements dans l'Histoire. Le style choisi par Pouqueville pour commencer son ouvrage reprend le rythme du début des Histoires. La référence au modèle' d'Hérodote est clairement exprimée :

«Avant que le temps ait effacé le souvenir des événements qui se sont passés dans la Grèce, depuis trois générations d'hommes, je veux essayer de les rapporter tels qu'ils sont venus à ma connaissance, afin que les souffrances des Hellènes, leurs mémorables actions et la barbarie des Turcs, puissent être connues du monde, occupé des événements dont l'Orient est le théâtre.

Cette tâche m'engage dans la narration d'une période qui a quelque similitude avec celle que les Muses d'Hérodote ont transmise à la postérité. Suivant de bien loin les traces du père de l'Histoire, je montrerai comment les Grecs, déchus de leur splendeur, subjugués par les Romains, qu'ils amollirent, dégradés sous le sceptre de leurs Césars théologiens, conquis par les Turcs, qu'ils ne purent civiliser, limant insensiblement leurs chaines, enveloppant le despotisme dans ses propres filets, s'emparèrent de l'héritage de la tyrannie et du crime, pour remonter au rang des nations " (Histoire..., p. 1-2).

La reconnaissance d'une identité grecque ${ }^{30}$, de l'existence d'une nation grecque, s'opère dans cette sorte de fusion des personnages et des événements de la Grèce ancienne et de ceux que connaît et que vit Pouqueville; représentation du croyant épris de culture antique (ils sont restés « Grecs " parce qu'ils étaient chrétiens) et regard du diplomate historien sur les choses politiques ${ }^{31}$.

29. Cf. Itinéraire, p. 36 et 37 : « Modon, où je foulai pour la première fois la terre sacrée des Hellènes, n'était pas l'arsenal des hordes d'Tbrahim; Navarin ne rappelait que Nestor et Pylos; Tripolizza [...] n'était pas un amas de décombres noircis par les flammes et dans lesquels tremble une garnison de bourreaux mahométans, disciplinée par des renégats chrétiens [...] Les restes des sculptures de Phidias n'avaient point encore été entassés pour servir d'abri à un peuple redevenu digne de camper dans ses remparts immortels. [...] La liberté n'avait point encore fait entendre le cri de sa renaissance du fond du tombeau d'Harmodius et Aristogiton. ")

30. Les Grecs modernes sont, dit Pouqueville « plus intéressants à étudier que la chronique de Paros ; car leur physionomie nationale tenait lieu d'inscriptions pour reconnaitre le passé et pour lire dans l'avenir : on y retrouvait les traits des Hellènes... " (Histoire..., p. 4).

31. La figure d'Ali Pacha est remarquable de ce point de vue. Ali de Tébélen, pacha de Jannina, " débuta à la manière des anciens héros de Grèce ". Personnage trouble, double, il prend successivement les aspects d'un conquérant moderne (dans sa ressemblance frappante 
Dans ce mouvement de la prise de conscience par l'intelligentsia française de l'existence d'une Grèce moderne, les références à l'Antique changent de registre. Ce ne sont plus les héros de la mythologie grecque qui apparaissent de façon signifiante (même si ces références demeurent) mais les grands personnages de la démocratie grecque, de l'histoire grecque. Harmodius et Aristogiton, les tyrannicides, libérateurs du peuple athénien opprimé plutôt que Nestor et Pylos pour Chateaubriand ; ou encore, pour Pouqueville, le vocabulaire si fortement marqué par la mythologie grecque s'inscrit dans le choix plus général et essentiel d'un récit historique à la manière du père de l'Histoire, Hérodote. Si l'on reconnait ainsi une nouvelle vie à la Grèce, vie qui s'exprime dans sa lutte armée $^{32}$ et dans sa renaissance culturelle ${ }^{33}$, faut-il pour autant ramener la Grèce moderne dans le camp des nations européennes parce qu'elle en a été la mère, la pensée civilisatrice qui les a fécondées? Ou faut-il reconnaître que la Grèce, telle que l'ont découverte les voyageurs, comme l'Égypte, est en Orient?

La question de la place à donner à la Grèce dans le monde contemporain est en fait au coeur des deux attitudes qui voient le jour au moment du soulèvement grec. Les deux " partis " (les « turcophiles » et les « philhellènes ") vont s'exprimer publiquement (dans les Chambres ou dans les journaux) sur la question d'une intervention française aux côtés des Grecs dans leur guerre contre les Turcs. Schématiquement ${ }^{34}$, les " turcophiles ", proches des milieux gouvernementaux, s'ils ne contestent somme toute pas l'aspect moral (dans le sens historique, la référence est à la Grèce ancienne) de la cause grecque, se prononcent toutefois contre toute aide ou intervention française au nom de raisons politiques et économiques. La Grèce appartient au gouvernement de la Porte, la

avec les actions du conquérant macédonien Alexandre) puis d'un despote oriental (ambitieux, cruel parce qu'il est un "Infidèle "). Alexandre Dumas s'est souvenu du livre de Pouqueville en écrivant Le Comte de Monte-Cristo : cf. éd. Paris, Livre de poche, 1964, t. 3, chap. LXxviı, " Haydée ", p. 35, où Ali Pacha apparaît par l'intermédiaire de sa fille.

32. Le soulèvement de 1821 , et les étapes de la lutte grecque semblent rythmer l'actualité européenne : 1822, les massacres de Chio; 1824, la mort de Byron à Missolonghi; 18251826, le long siège de Missolonghi qui s'achève par la chute de la ville le 20 avril ; 1827, la victoire navale de Navarin... Voir le travail de Jean Dimakis, La Presse française face da la chute de Missolonghi et à la bataille de Navarin : recherches sur les sources du philhellénisme français, Thessalonique, Institute for Balkan studies, vol. 162, 1976.

33. Adamantios Coray est lui-même un représentant de cette renaissance culturelle grecque, perceptible depuis la seconde moitié du xvire siècle (cf. son Mémoire sur l'état de la civilisation dans la Grèce, présenté à la Société des Observateurs de l'homme à Paris, le 6 janvier 1803). Ses traductions de textes antiques en grec moderne témoignent de son attachement à y faire participer ses compatriotes.

34. La prise de position vis-à-vis de la cause grecque apparaît avant tout comme une décision individuelle parce qu'elle appartient au domaine de la morale. Alfred de Vigny prend ainsi parti en faveur des Grecs dès 1822 , par le poème Héléna, contrairement aux tendances du mouvement politique auquel il appartient. 
révolte est donc illégitime, facteur de perturbations dans l'Europe conservatrice. D'autre part, les intérêts français (politiques et économiques) en Orient, et plus particulièrement en Égypte, alliée de la Porte dans la lutte contre les Grecs, risqueraient d'être mis à mal si la France prenait position en faveur de la Grèce. En fait, pour les diplomaties européennes, la Grèce fait partie de la question d'Orient et entre dans le jeu compliqué de l'équilibre des puissances. L'Europe du congrès de Vienne, de la SainteAlliance reste passive. Il faudra attendre Navarin (20 octobre 1827) pour qu'à la suite du traité de Londres ${ }^{35}$ les puissances interviennent directement dans le conflit aux côtés des Grecs; encore la solution politique envisagée pour la Grèce n'est-elle qu'une autonomie sous suzeraineté ottomane.

A cette Europe de la Sainte-Alliance, alliance des têtes couronnées, s'oppose ou répond le mouvement philhellénique, vaste mouvement européen (l'Amérique y participe également), qui se mobilise dès 1821 . Sorte de diplomatie parallèle (le Comité philhellénique de Paris entretient ainsi un délégué permanent auprès du gouvernement grec), le philhellénisme constitue tout un réseau de communication pour collecter et acheminer des fonds, vivres et armes aux combattants grecs et envoyer les volontaires européens sur place. La vie politique et culturelle de la France sera marquée durant les années de la lutte grecque par les manifestations diverses de ce philhellénisme : publication d'œuvres historiques, romanesques, poétiques ou picturales (Pouqueville, Lamartine, Delacroix...), concerts, expositions de peinture (les Salons), quêtes, appels, mode vestimentaire. Des séries d'assiettes prendront pour motif les combats des héros grecs et philhellènes ${ }^{36}$... Autant d'actions qui veulent entraîner l'opinion publique dans un soutien à la cause grecque et faire pression sur les cabinets européens pour les pousser à intervenir. Les motivations, comme les hommes qui composent ce mouvement, sont diverses. Il s'agit d'un devoir moral pour l'Occident d'aider cette Grèce qui lui a transmis son savoir à reprendre son rang parmi les nations européennes; devoir moral encore que d'aider des frères chrétiens - frères d'Orient - à

35. En avril 1827, la France, l'Angleterre et la Russie veulent imposer leur médiation dans le conflit, en vue de l'organisation d'une Grèce autonome dans le cadre de l'empire ottoman. La politique autrichienne de Metternich, hostile à toute intervention européenne, et favorable aux Turcs, était là battue en brèche. D'autant que les amirautés française et anglaise, interprétant le traité, transforment la simple interposition entre les forces ennemies en une intervention armée. La flotte turco-égyptienne, et quelques vaisseaux autrichiens qui l'accompagne, est détruite dans la rade de Navarin. Le retentissement de la "victoire " de Navarin est considérable en France. Chaque parti tentera, pour les élections qui ont lieu la même année, de s'attribuer le mérite du règlement de la question grecque.

36. Cf. Angélique AMANDRY, L'Indépendance grecque dans la faïence française du $x I X^{e}$ siècle, Athènes, Fondation ethnographique du Péloponnèse, 1982. 
combattre les Infidèles; devoir moral toujours, pour une nation qui a prôné la liberté des peuples à disposer d'eux-mêmes, d'aider un peuple à se libérer du joug d'un gouvernement étranger. Libéraux et ultras peuvent ainsi se rejoindre dans le soutien de la cause grecque.

Ce que révèlent ces deux attitudes vis-à-vis de la question grecque, c'est la tentative d'une définition des limites et du sens que peut prendre cette Europe face à cet Autre que représente l'Orient.

La campagne d'Égypte de 1798 prend, dans cette perspective, une importance capitale dans ce regard que l'Occident va, en retour, porter sur lui-même. C'est tout au moins la lecture que donnent régressivement les penseurs de l'événement qui nous importe ici. L'expédition de Bonaparte a trouvé historiquement et surtout symboliquement à Saint-Jean d'Acre sa limite ultime ${ }^{37}$. Campagne militaire et scientifique, les conséquences en sont considérables. Stratégiquement, le mouvement des conquêtes arrêté à l'Orient, se poursuivra en Occident, traçant les axes de cette Europe des nationalités. Scientifiquement et culturellement, les progrès de la connaissance de l'Égypte ancienne et moderne (Description de l'Égypte, déchiffrement des hiéroglyphes...) vont susciter un regain d'attention tant de la part du public que des scientifiques pour l'Orient. Avec l'Égypte, on reste cependant dans l'ancrage ou la sphère grecque ${ }^{38}$. Mais l'Orient c'est aussi l'Asie et surtout l'Inde. Le regard se déplace suivant le même itinéraire que s'est ouvert la Grèce antique avec Alexandre. Le travail sur les langues est en ce sens le plus frappant. Le début du $\mathrm{xIX}^{\mathrm{e}}$ siècle voit la naissance de cette linguistique historique fondée sur l'idée de l'évolution des langues et sur le fait que histoire des langues et histoire des peuples sont intimement liées. Les affinités des langues permettent de reconstituer les trajets historiques des peuples jusqu'à des origines communes $^{39}$. Ce que font apparaître les travaux sur les langues, et plus

37. C'est ainsi, par ex., qu'Alexandre Dumas, merveilleuse caisse de résonance, nous représente l'événement. Cf. Mes Mémoires, Paris, Gallimard, 1957, t. 1, p. 155 : « Bonaparte, après avoir échoué devant Saint-Jean d'Acre, voyait ses projets gigantesques sur l'Orient échouer devant une bicoque. " Ou encore dans Les Mohicans de Paris, Paris, Livre de poche, 1973, p. 418-420, l'extraordinaire dialogue de Napoléon et du tsar Alexandre écrit par Dumas.

38. En amont de l'expansion de la civilisation grecque, il y a l'Égypte et son influence en Grèce, plus particulièrement dans le domaine religieux. En aval, l'Égypte des Ptolémées inscrit son rayonnement culturel dans la tradition grecque. L'école d'Alexandrie rassemble les savants du monde hellénistique; mais dans ce mouvement vers l'Orient, ouvert par Alexandre, Athènes, comme centre, n'est pas déstabilisée. Cf. Bertrand Gille, Les Mécaniciens grecs. Naissance de la technologie, Paris, Seuil, 1980.

39. Le rapport à la langue (et non au langage, conception mécaniste du xviI' siècle) s'énonce différemment dans le contexte de la découverte de l'Autre, des nationalités. La langue est saisie comme quelque chose de vivant. Le travail sur la langue devient un travail sur l'origine ; il ne se pose plus à propos d'une culture ou du sentiment d'un destin commun, mais à propos d'une histoire. 
particulièrement l'ouvrage de Bopp ${ }^{40}$, c'est, par la grammaire comparée, la parenté existant entre le sanscrit, langue sacrée de l'Inde ancienne, et la plupart des langues européennes; une famille de langues, appelée " indo-européenne " ou " indo-germanique », dont la parenté serait due à l'existence d'une langue mère, l'indo-européen, dont elles seraient dérivées (cette langue n'est pas directement connue quoique les premiers comparatistes aient cru reconnaître dans le sanscrit cette langue mère). Cette découverte revêt aux yeux des contemporains, sensibilisés dans le contexte des nationalités au problème des langues, la preuve scientifique d'une parenté et d'une antériorité historique même de l'Inde sur les civilisations occidentales (renforcée par les autres recherches menées sur l'Inde ancienne : religion, littérature, arts). Avec la vision de l'Inde, berceau de la civilisation, on sort de la sphère grecque. La Grèce ancienne fait désormais partie d'un processus qui débute en Orient et dont la Perse, l'Égypte, la Grèce et Rome constituent les étapes. Ainsi, en même temps que les limites du monde moderne reculent, les limites de l'histoire sont repoussées dans le temps et dans l'espace ${ }^{41}$.

Mais la grandeur de l'Orient est passée, il est maintenant misérable, dégradé. Face à lui, l'Occident apparaît vif, fécond, en pleine expansion. Les nombreux voyages des penseurs à travers l'Europe ouvrent au mouvement des peuples et des idées, à la connaissance des cultures propres à chaque peuple, à chaque nation. La publication des Chants populaires grecs de Claude Fauriel en 1824 est très importante dans la perspective de la reconnaissance d'une culture populaire qui prend, dans le mouvement des nationalités, toute sa valeur et son sens. L'Occident découvre sa propre identité, sa propre civilisation - différence - face à l'Orient ${ }^{42}$. L'Europe, ce continent qui vient au monde, a sa propre histoire. Il cherche son origine dans le Moyen Âge, fond commun des différents pays qui le composent ; c'est le merveilleux chrétien, l'épopée, les sagas... Le centre géographique du nouveau continent se découvre, dans un mouvement de translation vers le Nord : ce sera l'Allemagne, "l'Inde de

40. Système de conjugaison de la langue sanscrite, comparé à celui des langues grecque, latine, persane et germanique, Francfort, 1816. C'est à Paris que Bopp a appris les langues orientales dans le cadre de l'École des langues orientales, fondée par le très actif Silvestre de Sacy (1755-1838). Cf. Raymond SchwaB, La Renaissance orientale, Paris, Payot, 1950.

41. Cf. Victor Hugo, préf. aux Orientales, 1829, Paris, Gallimard, 1964, p. 24 : « Ne verrait-on pas plus haut et plus loin, en étudiant l'ère moderne dans le Moyen Âge et l'Antiquité dans l'Orient. "

42. La reconnaissance d'une Europe des spécificités des langues, des spécificités politiques, par la médiation de l'Ancien (comme transparent à l'histoire) et à travers l'existence d'une langue matricielle, l'indo-européen, découvre une limite symbolique qui croise les limites géographiques de l'Europe (Orient/Occident) circonscrites par la campagne de 1798. 
l'Europe ${ }^{43}$. De cette Allemagne doit s'épanouir la nouvelle civilisation, comme l'Inde avait été le point d'origine de l' " ancienne".

La Grèce, dans ce mouvement, ne disparait pas; elle reste le fondement le point ultime de référence par rappost auguei on se situe. II fallait ce détachement de la Grèce mère par la découverte d'une Grèce orientale (c'est-à-dire misérable), la découverte d'une autse source plus proche, enracinée dans une terre (le Moyen Âge), la découverte enfin d'une Antiquité plus lointaine et plus vaste (l'Inde), pour parvenir à dépasser l'image figée de cette « Grèce qu'on connaît trop » pour reprendre Hugo. La Grèce n'apparaît plus comme une Antiquité figée, un pays mort enseveli sous ses ruines si belles. Le modèle grec, comme celui de l'épanouissement d'une civilisation, peut alors jouer pleinement son rôle. Il faut non pas imiter (la Grèce ancienne a retrouvé sa place dans l'histoire, comme passé révolu), mais transposer, comprendre, faire sien ce modèle. Il faut faire revivre l'Antiquité en l'homme dans ce qu'elle avait de plus profond, de plus grand, dans son principe même ; une grande civilisation féconde dans tous les domaines et qui s'est perpétuée, ouvrant à la réconciliation du moi et de l'Histoire, des individus et de la nature - un cosmos. Les anciens Grecs revivent par les hommes du ternps présent qui en portent le savoir ou l'esprit. Par le pouvoir d'évidence de l'èvénement, l'Ancien bascule dans le Nouveau ${ }^{44}$. L'Antiquité est en l'homme qui se libère ainsi du poids du passé et en fait une réalité présente, comme les Grecs modernes qui luttent pour affirmer leur identité et leur indépendance. La Grèce est un pays neuf aussi, "libre de toute Antiquité », disait Pouqueville.

Derrière un modèle d'explication aussi tranchè que Grèce ancienne, idéale, mythicue, et Grèce moderne, politioue, se cache en fait la prise de conscience d'une époque, d'une nationalité, d'us tessitoire; la découverte d'une liberté, celle de l'individu, de l'homme. Le voyageur de l'Amérique et de l'Orient, Chateaubriand, peut ainsi encore s'écrier: "Quoiqu'il arrive, je mourrai Grec. "

Marie-Pascale Macia-WidemanN.

43. Cette expression revient fréquemment dans les textes de l'époque Rappelons ce que disait $\mathbf{M}^{\mathrm{me}}$ de Staël, De l'Allemagne, Paris, Garnier-Flammarion, 1968, t. 1, p. 41 : “ L'Allemagne, par sa situation géographique, peut être considérée comme le cour de l'Europe, et la grande association continentale ne saurait retrouver son indépendance que par celle de ce pays." "

44. Pascal, Euvres complètes, Préface sur le traité du vide, Paris, Seuil, 1963, p. 232 : "Ceux que nous appelons anciens étaient véritablement nouveaux en toutes choses, et formaient l'enfance des hommes proprement; comme nous avons joint a leurs connaissances l'expérience des siècles qui les ont suivis, c'est en nous qu'on peut trouver cette antiquité que nous révérons dans les autres. 》 